\title{
Health-related quality of life in pregnancy with uterine fibroid: a cross-sectional study in China
}

\author{
Wai-Kit Ming ${ }^{1,2^{*}}$, Huailiang $\mathrm{Wu}^{1,3 \dagger}$, Yanxin $\mathrm{Wu}^{1 \dagger}$, Hanqing Chen ${ }^{1}$, Tian Meng ${ }^{1,3}$, Yiwei Shen ${ }^{1,3}$, Ziyu Wang ${ }^{1,3}$, \\ Xinyu Huang ${ }^{1,3}$, Weiwei Sun ${ }^{1,3}$, Tik Sang Chow ${ }^{1,3}$, Yuan Wang ${ }^{1,3}$, Wenjing Ding ${ }^{1}$, Haitian Chen ${ }^{1}$, Zhuyu Li \\ and Zilian Wang ${ }^{1 *}$
}

\begin{abstract}
Background: Uterine fibroids (UFs) are the most common benign tumors in women. They are likely to cause numerous clinical symptoms, such as pain, menorrhagia, and other obstetric complications in pregnant women. This study aimed to determine the health-related quality of life (HRQoL) during pregnancy with uterine fibroids (UF) , thus providing a utility-based case value in pregnant women with UF and understanding of whether HRQoL is associated with clinical outcomes in pregnant women with UFs.

Method: This study was conducted in a cross-sectional manner. This study was based on questionnaire surveys completed by sequential out- and in-patients and was conducted in a regional university hospital in Guangzhou, China. The EuroQoL five-dimension-five-level (EQ-5D-5 L) questionnaire was used, and demographic data were collected. An electronic record of the clinical outcomes of pregnant women with UF was retrieved from the hospital's electronic medical record system. The association between UF and HRQoL was evaluated by ordered regression.

Results: Seven-hundred-sixty-seven pregnant women with a mean age (SD) of 32.7 (4.8) years completed 707 questionnaires. Overall, when comparing the UF with non-UF groups, we detected statistical differences in age, body mass index (BMI), gravidity and abortion times, partner's smoking and alcoholic habits, advanced maternal age, and uterine scars $(p<0.05)$. Furthermore, pregnant women without UF scored significantly higher than those with UF on the EQ-5D value system $(0.84$ versus $0.79 ; p=0.017)$. Moreover, pregnant women with UF suffered more health-related problems, especially with respect to self-care (odds ratio $[O R]=3.69, p<0.01$ ) and usual activity dimensions $(O R=2.11 ; p=0.01$ ).

Conclusion: We found that UF has a negative impact on the HRQoL of pregnant women with respect to self-care and usual activity dimensions. Also, the EQ-5D score was a better index than the EQ-VAS score for HRQoL when evaluating of the QoL of our population of pregnant women.
\end{abstract}

\section{Introduction Background}

Uterine fibroids (UFs), also known as uterine myomas, fibromyomas, or leiomyomatas, are the most common benign tumors in women and have clinical morbidity rates of 20 to $40 \%$ and prevalence rates of 3 to $12 \%$ during pregnancy. The common causes of UFs are variable

\footnotetext{
*Correspondence: mingwj@mail.sysu.edu.cn; wkming@connect.hku.hk; wangzil@mail.sysu.edu.cn

${ }^{+}$Wai-Kit Ming, Huailiang Wu and Yanxin Wu contributed equally to this work 'Department of Obstetrics and Gynaecology, The First Affiliated Hospital of Sun Yat-sen University, Guangzhou, China

Full list of author information is available at the end of the article
}

factors, such as genetics, endocrine factors and lifestyle factors [1-3]. The clinical outcomes range from asymptomatic to presentation of pain, menorrhagia, and obstetric complications such as infertility, miscarriage, and/ or scarred uterus $[3,4]$. However, these symptoms can affect the quality of life for pregnant women [5].

In a recent study, pregnant women were shown to suffer from an increase in the risk of depression [6], and depressive symptoms correlate with impairment of HRQoL [7]. According to prior studies, pain was shown to be the most common symptom of UF during pregnancy, and the risk of depression could increase in this

(c) The Author(s). 2019 Open Access This article is distributed under the terms of the Creative Commons Attribution 4.0 International License (http://creativecommons.org/licenses/by/4.0/), which permits unrestricted use, distribution, and reproduction in any medium, provided you give appropriate credit to the original author(s) and the source, provide a link to the Creative Commons license, and indicate if changes were made. The Creative Commons Public Domain Dedication waiver (http://creativecommons.org/publicdomain/zero/1.0/) applies to the data made available in this article, unless otherwise stated. 
situation [3, 8]. Some past studies used the EuroQoL Group's five-dimension questionnaire (EQ-5D) to measure and assess the relationship between pain, depressive symptoms, and quality of life (QoL) [7, 9, 10]. Considering that most pregnant women do not have fatal diseases, it was suitable to use a generalized questionnaire to assess their HRQoL values. Therefore, as one of the most common instruments for measuring the overall body health state, the EQ-5D is a powerful and popular tool, especially for assessing pain and anxiety/depression symptoms [11, 12]. The symptoms and clinical outcomes caused by UFs might correlate with HRQoL; therefore, clinicians can use HRQoL as an index to evaluate the effectiveness of treatment $[13,14]$.

In contrast to traditional clinical outcomes, HRQoL during pregnancies with UFs can be used as an outcome indicator in health policy research, facilitating the improvement of clinical UF management. Utility data are key factors for cost-utility analysis and quality-adjusted life-year analysis in healthcare-related economics but have not been addressed in the literature; therefore, this study could provide a utility-based case value in pregnancies with UFs.

\section{Objectives}

We aimed to evaluate several parameters: (1) determine the HRQoL in pregnancies with UFs; (2) provide a utility-based case value in pregnancies with UF; and (3) understand whether HRQoL is associated with the clinical outcomes of pregnant women with UFs.

\section{Method}

\section{Study design}

A cross-sectional study was performed as a part of a longitudinal project that studied pregnant women who received prenatal care during different gestational ages at one of the largest regional university hospitals in south China (The First Affiliated Hospital of the Sun Yat-sen University) from May 2017 to February 2018. Ethical approval was granted by the Institutional Review Board of The First Affiliated Hospital of Sun Yat-sen University (ICE-2017-296). All procedures were conducted in accordance with the Declaration of Helsinki. All participants signed the informed consent documents before participation in this study.

\section{Study population}

All participants came from The First Affiliated Hospital of the Sun Yat-sen University. Eligible participants were included if they were pregnant. Only the first record for each participant was included in this study. Participants were excluded when they had missing personal information and/or clinical data. Furthermore, if any participants completes more than one EQ5D questionnaire, all additional records were excluded, except for the first one.

\section{Measurement}

Patient-evaluated HRQoL is an important index in the assessment of a patient's health and functional states [15]. The EuroQoL Group's five-dimension questionnaire (EQ-5D) with EuroQoL Group's visual analog scale questionnaire (EQ-VAS) is a common questionnaire for measuring the quality of life, making cost-efficiency calculations, and evaluating economic issues in the public health field. The EuroQoL Group's five-dimension five-level questionnaire (EQ-5D-5 L) is a more reliable and sensitive instrument for measuring HRQoL than the EQ-5D-3 L [16]. The EQ-5D-5 L instrument contains a descriptive system for assessing a participant's health state over five dimensions based on five levels in each dimension and utilizes a self-determined visual analog scale (VAS). These two parts were used throughout this study. The Chinese version of the EQ-5D-5 L has been shown to be valid and effective and is commonly used to measure HRQoL [17, 18]. EQ-VAS can provide a self-reported global measure of overall health and broader dimensions of assessment than EQ-5D although more than half the participants do not accurately evaluate themselves when using these types of scoring systems $[19,20]$. This study might help to identify which of these two independent tools is more suitable for assessing pregnant women with UFs and provide detailed HRQoL data for future studies.

Participants were administered the EQ-5D questionnaire the first time that they visited the hospital for prenatal care. The EQ-5D assessed five dimensions (mobility, self-care, usual activity, pain/discomfort, and anxiety/depression) and it was based on five problem levels: (1) none; (2) slight; (3) moderate; (4) severe; and (5) extreme/unable. As examples, the self-care dimension asks about the degree of problems experienced when "washing and dressing by yourself", and the usual activity dimension asks about the degree of problems in "work, study, housework, family, or leisure activities in daily life". The five levels of response were represented by integer values (such as $1-5$ with values of $2-5$ indicating health-related problems) $[16,17,19]$. Each response pattern was calculated into a single EQ-5D index value (such as 11,221) through the EQ-5D-5 L Crosswalk Index Value Calculator to produce a final QoL value. The value ranged from -0.224 to 1 with 1 indicating the best health state of people, whereas 0 represents death. Most patients are in the range from 0 to 1 ; however, it is still possible to achieve scores $<0$, and these negative values correspond with overall health states (both physical and mental) that are considered worse than death [18]. We then measured each dimension and compared 
responses between pregnant women with and without UF. The EQ-VAS was a self-assessment of health state across five dimensions based on five levels of response. It presented as a vertical line with demarcations from 100 (best imaginable health state) to 0 (worst imaginable health state) [21]. Respondents were asked to draw a line from the bottom line 0 to the score line based on their opinion of their health states and fill the score in the blank beside.

\section{Variables}

Basic independent covariates of the study population in the models included age, body mass index (BMI), living location, gravidity, parity, abortion, gestational trimester (first, second, or third), and advanced maternal age (indicated the age of pregnant women $>35$ years old) [22] A meta-analysis showed no significant impact of smoking on risk of UFs [23]. However, we wanted to detect whether the smoking state of pregnant women could affect the HRQoL in those pregnant women with UF. Furthermore, the partners' lifestyle habits, such as smoking status and alcohol consumption, were also included in this study. Also, multipara, uterine scar, hepatitis B history, heart disease, and surgical history were included as part of the index for pre-pregnancy conditions. Throughout the study, participants were categorized by gestational trimester in which the first trimester was taken when the pregnant women were at the gestational age of $<13$ weeks, the second was the gestational age of 13 to 28 weeks, and the third was a gestational age $>28$ weeks. In generalized situations, we use transvaginal ultrasonography to detect and diagnose uterine fibroids. However, on rare occasions, such as suspected carcinoma (indicated by elevated cancer biomarker levels), a pathological examination might need to be performed to distinguish the uterine fibroids from uterine carcinoma under the current guideline in our hospital. In this situation, the risk of miscarriage due to the procedure needs to be balanced [24]. In this study, we did not have any cases that needed to undergo pathological examinations.

\section{Bias}

The EQ-5D questionnaire was a subjective measurement of pregnant women's HRQoL, and self-reported bias may be the main bias in this study. Based on the population, this study also minimized selection bias but had non-response, volunteer, and ascertainment biases.

\section{Statistical methods}

Data analysis was performed using the STATA/SE 14.0 for Windows. Normally distributed continuous variables were described using the means + standard deviations (SDs), and ranges. Non-normal variables were presented as the median, and categorical variables were described using counts and percentages. The dependent variables were the EQ-5D score utility and EQ5D-VAS. EQ-5D scores were in a skewed distribution; therefore, we used a non-parametric approach to analyze the data.

Participants' demographic data were reported (age, advanced maternal age, BMI, local, gravity, party, abortion, smoking, partner smoking status and alcoholic consumption, multipara, surgery history, hepatitis B, and heart disease). The clinical outcomes were retrieved from the hospital electronic medical system after delivery. Since the EQ5D values present a skewed distribution, we divided these values into two groups based for statistical analysis on the median EQ5D values: (1) above the median and (2) below the median [25]. Analysis of variance and $\mathrm{t}$ - and the chi-squared tests were used to compare continuous and qualitative variables among the three different trimesters. Health quality, as measured by the EQ5D-VAS scores or EQ5D values, and multiple linear regressions was used. Potential confounders were adjusted. An ordered logistic regression with odds ratios (ORs) and 95\% confidence intervals (CIs) is an appropriate method to use when examining the effects of independent risk factors on various dimensions in the EQ5D index when complementary dimensions are taken into account $[26,27]$. ORs, 95\% CIs, and $p$-values were obtained using an ordered logistic regression analysis. All tests were two-sided, and a p-value of 0.05 was considered as statistically significant.

\section{Results}

\section{Participants}

In total, all 767 pregnant women agreed to participate in this study, but of these 60 were excluded due to missing clinical data or personal information. We only reserved the first record as their HRQoL. Therefore, 707 of the included pregnant women provided 707 EQ-5D-5 L valid questionnaires for the analysis. Using electronic medical records, we identified 105 pregnant women with UFs. Of these, 103 were included in the study because two women did not deliver within the period under analysis (Fig. 1).

\section{Descriptive data \\ General characteristics of patients}

Of 707 pregnant women, the ages ranged from 21.3 to 47.4 years, and the mean age was $32.7 \pm 4.8$ years. Of those, in UF group, 105 women (14.9\%) were included and were $35.6 \pm 4.8$ years on average, while the non-UF group of pregnant women's mean age was 32.3 (4.5) years (Table 1). The mean BMI across those with UFs was $26.4 \pm 4.0$ and was significantly lower $(23.5 \pm 3.9)$ among those without UFs.

Of the 707 eligible pregnant women, most (90.9\%) lived locally (living in Guangzhou). The mean values for 
All 767 agreed to participate in the study

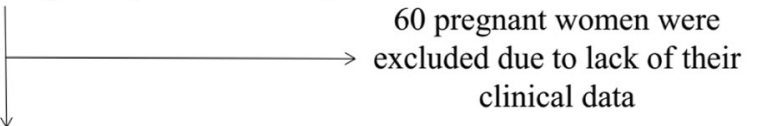

707 pregnant women with 1043 EQ-5D records

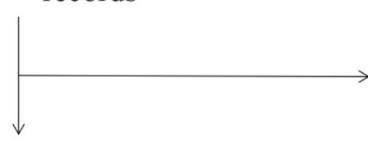

707 pregnant women with 707 EQ-5D records were included and 105 women had uterine fibroids

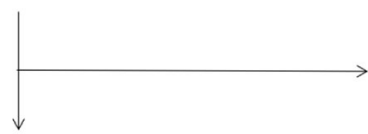

103 clinical outcomes of pregnant women with uterine fibroids
336 EQ-5D records were excluded because we only reserved the first EQ-5D recods as their health-quality of life values

2 pregnant women hadn't reached delivery

Fig. 1 Selection of the study population

gravidity, parity, and abortion times across the entire population were $2.2 \pm 1.1,0.5 \pm 0.5$, and $0.6 \pm 0.9$, respectively. All 707 pregnant women denied smoking during pregnancy. Some women's partners (15.4\%) were smokers, and $8.1 \%$ of the partners consumed alcohol. Fifteen percent of the sample population was multipara, $24.8 \%$ had surgical histories, $30.3 \%$ were of advanced maternal age, $23.2 \%$ had scars on the uterus, $2.1 \%$ had hepatitis $\mathrm{B}$, and $0.9 \%$ had chronic heart disease. Table 1

Table 1 Demographic characteristics

\begin{tabular}{lllll}
\hline & UF & $\begin{array}{l}\text { Non-UF } \\
(n=105)\end{array}$ & $\begin{array}{l}\text { All patients } \\
(n=602)\end{array}$ & $p$-value \\
\hline Age (SD) & $35.6(4.8)$ & $32.3(4.5)$ & $32.7(4.8)$ & $<\mathbf{0 . 0 1}$ \\
Advanced maternal age & $53(50.5 \%)$ & $161(26.7 \%)$ & $214(30.3 \%)$ & $<\mathbf{0 . 0 1}$ \\
BMI (SD) & $26.4(4.0)$ & $23.5(3.9)$ & $23.9(4.1)$ & $<\mathbf{0 . 0 1}$ \\
Living location & $96(91.4 \%)$ & $547(90.9 \%)$ & $643(90.9 \%)$ & 0.85 \\
(Guangzhou) & & & & \\
Gravidity (SD) & $2.4(1.2)$ & $2.1(1.1)$ & $2.2(1.1)$ & $\mathbf{0 . 0 3}$ \\
Parity (SD) & $0.6(0.5)$ & $0.5(0.5)$ & $0.5(0.5)$ & 0.57 \\
Abortion (SD) & $0.8(0.9)$ & $0.6(0.8)$ & $0.6(0.9)$ & $\mathbf{0 . 0 1}$ \\
Smoking & $0(0.0 \%)$ & $0(0.0 \%)$ & $0(0.0 \%)$ & - \\
Partner Smoking & $24(22.9 \%)$ & $85(14.1 \%)$ & $109(15.4 \%)$ & $\mathbf{0 . 0 2}$ \\
Partner Alcoholics & $14(13.3 \%)$ & $43(7.1 \%)$ & $57(8.1 \%)$ & $\mathbf{0 . 0 3}$ \\
Multipara & $5(4.8 \%)$ & $101(16.8 \%)$ & $106(15.0 \%)$ & $<\mathbf{0 . 0 1}$ \\
Surgical history & $26(24.8 \%)$ & $149(24.8 \%)$ & $175(24.8 \%)$ & 1.00 \\
Uterine scar & $33(31.4 \%)$ & $131(21.8 \%)$ & $164(23.2 \%)$ & $\mathbf{0 . 0 3}$ \\
Hepatitis B & $1(1.0 \%)$ & $14(2.3 \%)$ & $15(2.1 \%)$ & 0.37 \\
Heart disease & $1(1.0 \%)$ & $5(0.8 \%)$ & $6(0.9 \%)$ & 0.90 \\
\hline UF & &
\end{tabular}

UF Uterine fibroids, BMI Body mass index. Bold represented $p$ value $<0.05$. Data with SD indicates "mean" value. Data without SD indicates "number" shows these risk factors for the sample population. Significant differences were found in age between those with and without UFs $(p 0.01)$, BMI $(p<0.01)$, gravidity $(p=0.03)$, abortion $(p=0.01)$, partner smoking $(p=0.02)$, partner alcoholism $(p=0.03)$, scarred uterus $(p=0.03)$, multipara $(p<0.01)$, and advanced maternal age $(p<0.01)$.

\section{Outcome data}

The characteristics of clinical outcomes in pregnant women with UFs

Two of the study participants did not deliver at the time point of analysis. Thus, these results only included clinical outcomes of $98.15 \%$ (103 out of 105) pregnant women with UFs. Among those pregnant women enrolled in the study (except for the two that had not delivered), about $44.7 \%$ belonged to the below median EQ-5D score group while $55.3 \%$ came from the above median EQ-5D score group (Table 2). The mean \pm SD gestational ages for the below median and above median EQ-5D score groups were $37.8 \pm 1.7$ and $38.0 \pm 1.6$ weeks, respectively. For pregnancy complications, no statistical difference was found between two groups.

\section{Main results}

The EQ-5D and EQ-VAS values assessed using the EQ-5D-5 L The EQ-5D and EQ-VAS value distributions are shown (Figs. 2 and 3). Of the total 707 EQ-5D and EQ-VAS records (707 pregnant women), the mean (SD) EQ-5D indices for those with and without UFs were $0.79 \pm 0.21$ and $0.84 \pm 0.18$, respectively $(p=0.017)$ and the mean of EQ-VAS with and without UFs were 88.0 \pm 8.6 and $87.3 \pm$ 9.9 , respectively $(p=0.480)$ (Table 3$)$. Besides, the groups also showed differences in age and BMI (both $p<0.01$ ) (Table 1). Therefore, age and BMI were adjusted in the 
Table 2 Clinical outcomes of pregnant women with uterine fibroids

\begin{tabular}{|c|c|c|c|c|}
\hline & $\begin{array}{l}\text { Below median } \\
\text { EQ5D score } \\
(n=52)\end{array}$ & $\begin{array}{l}\text { Above median } \\
\text { EQ5D score } \\
(n=51)\end{array}$ & $\begin{array}{l}\text { All UF patients } \\
(n=103)\end{array}$ & $p$-value \\
\hline Cesarean Section & $39(75.0)$ & $31(60.8)$ & $70(68.0)$ & 0.91 \\
\hline Preterm labor & $5(9.6)$ & $9(17.6)$ & 14(13.6) & 0.67 \\
\hline Precipitate labor & $0(0.0)$ & $3(5.9)$ & $3(2.9)$ & 0.11 \\
\hline Placenta adherence & $8(15.4)$ & $7(13.7)$ & 15(14.6) & 0.34 \\
\hline Nuchal cord around neck & 10(19.2) & 15(29.4) & $25(24.3)$ & 0.19 \\
\hline PROM & $11(21.2)$ & $15(29.4)$ & $26(25.2)$ & 0.28 \\
\hline Postpartum hemorrhage & $1(1.9)$ & 2(3.9) & $3(2.9)$ & 0.44 \\
\hline Amniotic fluid turbidity & $6(11.5)$ & $6(11.8)$ & $12(11.7)$ & 0.31 \\
\hline Perineal laceration & $7(13.5)$ & 10(19.6) & $17(16.5)$ & 0.83 \\
\hline Hypertensive disorders & $14(26.9)$ & $9(17.7)$ & $23(22.3)$ & 0.90 \\
\hline Gestation age at birth & $37.8(1.4)$ & $38.1(1.9)$ & $37.9(1.7)$ & 0.73 \\
\hline Apgar score - 1 min & $9.85(0.5)$ & $9.75(0.63)$ & $9.8(0.57)$ & 0.13 \\
\hline Fetal distress & $5(9.6)$ & $12(23.5)$ & $17(16.5)$ & 0.07 \\
\hline
\end{tabular}

PROM Premature rupture of membrane

analysis. After adjustment, EQ-5D scores were significantly different between women with and without UFs ( $p$ $=0.007$ ), while the EQ-VAS score showed no statistically significant difference between the two groups $(p=0.486)$.

The EQ-5D and EQ5D-VAS scores varied across the different gestational trimesters (Figs. 4 and 5). Pregnant women with UFs scored lower indices on the EQ-5D compared to those without UFs, regardless of the trimester. Among those without UFs, the mean EQ5D indices were $0.75,0.88$, and 0.82 in the first, second, and third trimesters, respectively. In the first, second, and third trimesters, mean EQ5D indices were 0.56, 0.83, and 0.78 , respectively, among those pregnant women with UF. The mean EQ5D-VAS scores were lower among those with UFs compared to those without, except in the third trimester. Also, women from both groups (non-UF versus UF) presented the greatest EQ-5D indices (0.88 versus 0.83 ) and EQ5D-VAS scores (88.1 versus 88.0 ) in the second trimester.

\section{Uterine fibroids and other factors contributing to health quality}

The 707 EQ-5D records were classified based on the presence of UFs in the participant. Table 4 shows that

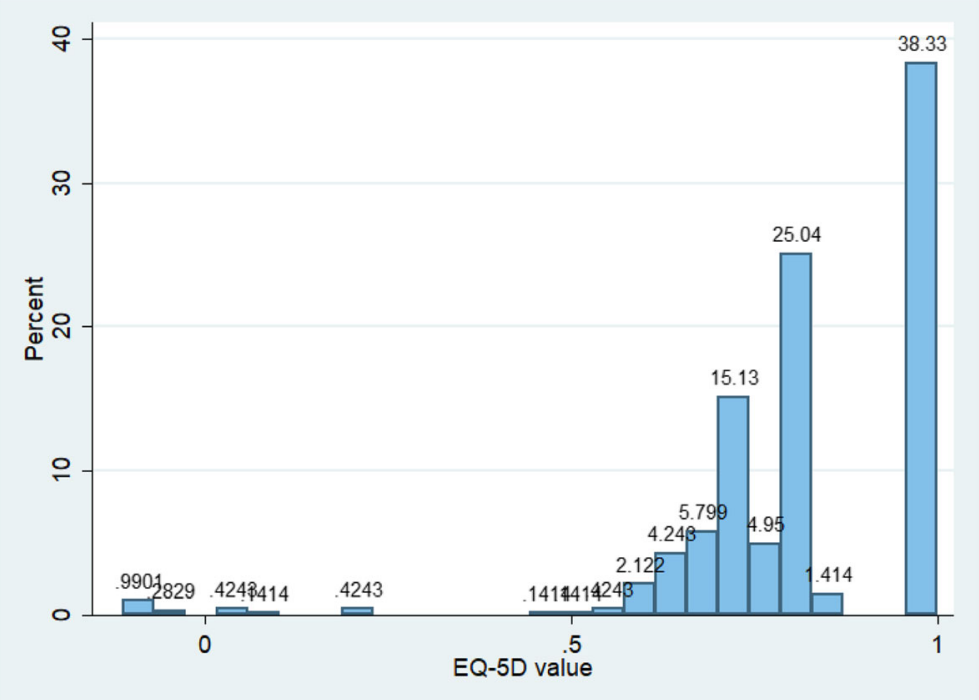

Fig. 2 Distribution of EQ-5D values of all pregnant women 


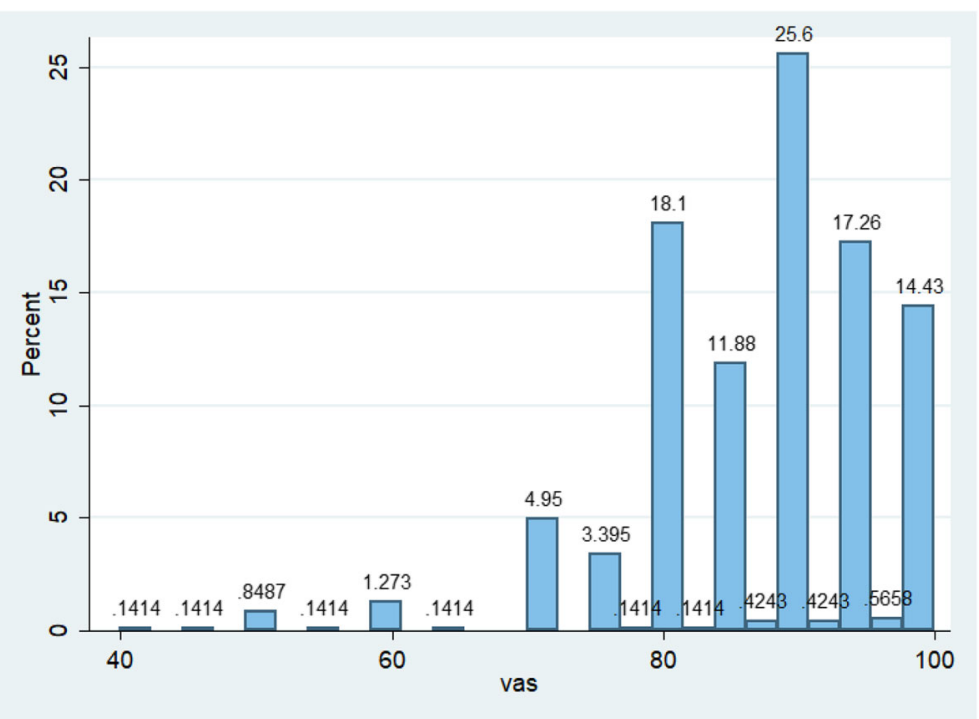

Fig. 3 Distribution of EQ5D-VAS values of all pregnant women

$23.8 \%$ of the records for those with UFs also indicated problems with mobility, $20.0 \%$ with self-care, $29.5 \%$ with usual activity, 52.4\% with pain/discomfort, and 35.2\% with anxiety/depression. Additionally, $18.9 \%$ of the pregnant women without UFs suffered mobility problems, $7.3 \%$ had self-care problems, $16.0 \%$ had problems with their usual activities, $45.7 \%$ had pain/discomfort problems, and $30.1 \%$ had anxiety/depression problems. The results indicate that pain and discomfort during pregnancy were major problems for pregnant women, while problems with self-care were of the least concern. Besides, there were about $13.5 \%$ more health-related problems with respect to the usual activity dimension in the UFs group than that in non-UFs group, which was a noticeable difference between pregnant women with and without UFs for the five dimensions. For the overall status, there was a greater proportion of those with UFs

Table 3 EQ-5D Index and EQ-VAS scores with and without UF EQ5D Index $(n=707)$

Unadjusted Age adjust Age \& BMl adjusted

\begin{tabular}{llll} 
UF group & $0.79(0.21)$ & $0.79(0.00)$ & $0.79(0.00)$ \\
Non-UF group & $0.84(0.18)$ & $0.84(0.00)$ & $0.84(0.00)$ \\
$p$-value & 0.017 & 0.002 & 0.007 \\
& EQ5D-VAS $(n=707)$ & \\
& Unadjusted & Age-adjusted & Age \& BMl adjusted \\
UF group & $88.0(8.6)$ & $88.0(0.01)$ & $88.0(0.01)$ \\
Non-UF group & $87.3(9.9)$ & $87.2(0.00)$ & $87.3(0.00)$ \\
$p$-value & 0.480 & 0.522 & 0.486 \\
\hline
\end{tabular}

UF Uterine fibroids, BMI Body mass index who experienced health-related problems (regardless of the dimensions) when compared to those without UFs.

An ordered logistic regression analysis was used for each dimension in the EQ-5D (Table 5). Women in their second or third trimesters reported more problems with mobility $(\mathrm{OR}=1.77 ; p<0.01)$ and pain/discomfort (OR $=1.46 ; p<0.01)$ than those in their first trimester. UFs were related to self-care problems $(\mathrm{OR}=3.69 ; p<0.01)$ and usual activity problems $(\mathrm{OR}=2.11 ; p<0.01)$.

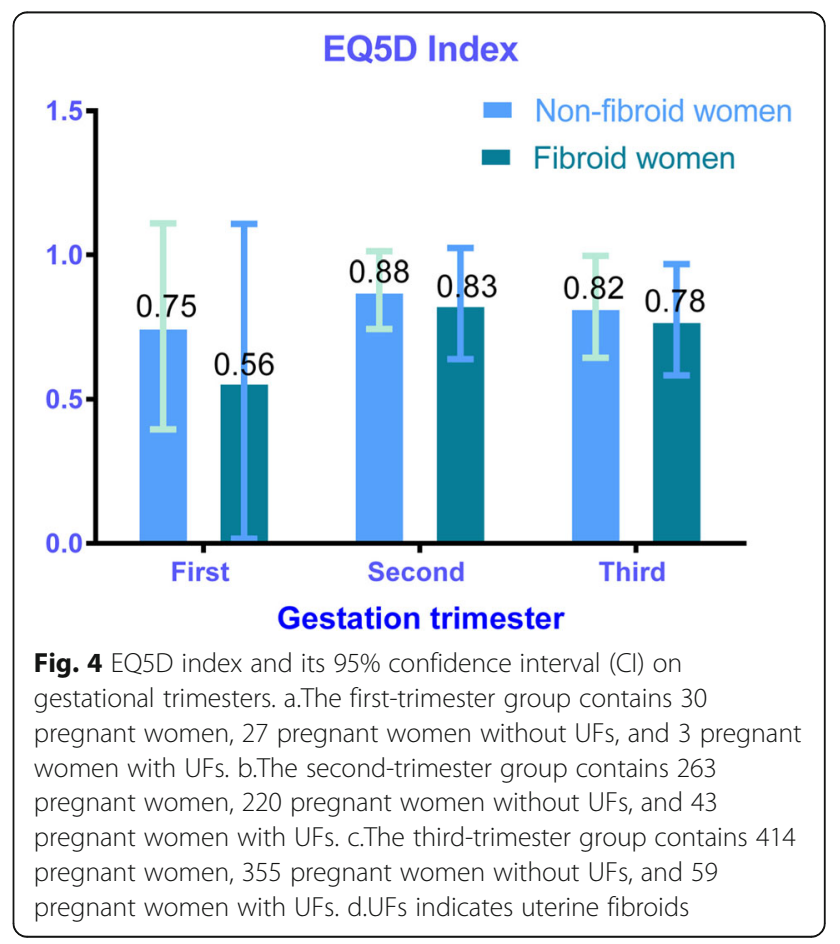




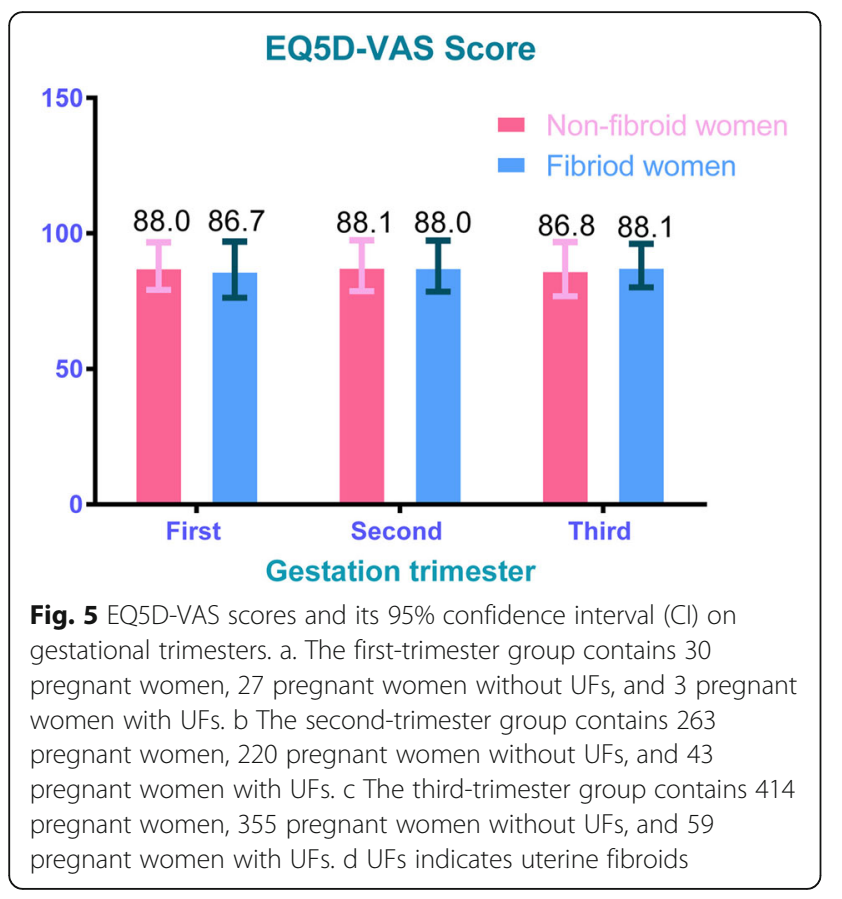

Pregnant women who were not located locally suffered from more severe pain/discomfort problems during the first trimester $(\mathrm{OR}=2.16 ; p<0.01)$. More gravidity was associated with problems regarding usual activity $(\mathrm{OR}=$ $1.33 ; p=0.01$ ), and less parity was associated with problems with usual activity $(\mathrm{OR}=0.56 ; p=0.03)$ and anxiety $/$ depression $(\mathrm{OR}=0.60 ; p=0.02)$.

\section{Discussion}

Key results

The major finding of the present study indicated that uterine fibroids could significantly affect HRQoL of pregnant women in two dimensions (self-care and usual activities) when compared to women without UF. Pregnant women with UFs had a lower EQ-5D index than those without UFs $(0.80$ versus 0.84$)$ while their average EQ5D-VAS scores were 88.0 versus 87.3 . This EQ-VAS result was similar to that found in other research in China in which evaluated health status in a similarly-aged cohort was evaluated [28, 29]. Less than $15 \%$ of pregnant women rated their health status as 100 (best possible health state) based on the EQ5D-VAS. However, the EQ-VAS scores did not show statistically significant differences between pregnant women with and without UFs, and the different standards of health self-evaluation from each participant could cause bias in the EQ5D-VAS results; thus, the EQ5D index might be a more suitable index than the EQ-VAS for evaluating HRQoL in pregnant women with UFs. Furthermore, pregnant women showed the greatest EQ-5D indices and EQ5D-VAS scores during their second gestational trimester
Table 4 The frequencies of pregnant women that report levels 1 to 5 for the various dimension

\begin{tabular}{|c|c|c|c|c|}
\hline \multicolumn{2}{|l|}{ EQ-5D Dimension } & \multicolumn{2}{|c|}{ Uterine fibroid } & \multirow[t]{2}{*}{ Total (\%) } \\
\hline & & $\overline{U F}(\%)$ & Non-UF (\%) & \\
\hline \multirow[t]{5}{*}{ Mobility } & Level 1 & $80(76.2)$ & $488(81.1)$ & $568(80.3)$ \\
\hline & Level 2 & 20(19.1) & $91(15.1)$ & $111(15.7)$ \\
\hline & Level 3 & $1(1.0)$ & $9(1.5)$ & $10(1.4)$ \\
\hline & Level 4 & $O(0.0)$ & $3(0.5)$ & $3(0.4)$ \\
\hline & Level 5 & $4(3.8)$ & $11(1.8)$ & $15(1.4)$ \\
\hline \multirow[t]{5}{*}{ Self-care } & Level 1 & $84(80.0)$ & $558(92.7)$ & $642(90.8)$ \\
\hline & Level 2 & $17(16.2)$ & $29(4.8)$ & $46(6.5)$ \\
\hline & Level 3 & $1(1.0)$ & $2(0.3)$ & $3(0.4)$ \\
\hline & Level 4 & $0(0.0)$ & $1(0.2)$ & $1(0.1)$ \\
\hline & Level 5 & $3(2.9)$ & $12(2.0)$ & $15(2.1)$ \\
\hline \multirow[t]{5}{*}{ Usual Activity } & Level 1 & $74(70.5)$ & $505(84.0)$ & 579(81.9) \\
\hline & Level 2 & $26(24.8)$ & $75(12.5)$ & $101(14.3)$ \\
\hline & Level 3 & $2(1.9)$ & $11(1.8)$ & $13(1.8)$ \\
\hline & Level 4 & $0(0.0)$ & $3(0.5)$ & $3(0.4)$ \\
\hline & Level 5 & $3(2.9)$ & $8(1.3)$ & $11(1.6)$ \\
\hline \multirow[t]{5}{*}{ Pain/Discomfort } & Level 1 & $50(47.6)$ & $327(54.3)$ & $377(53.3)$ \\
\hline & Level 2 & $49(46.7)$ & $246(40.9)$ & 295(41.7) \\
\hline & Level 3 & $3(2.9)$ & $15(2.5)$ & $18(2.6)$ \\
\hline & Level 4 & $0(0.0)$ & $6(1.0)$ & $6(0.9)$ \\
\hline & Level 5 & $3(2.9)$ & $8(1.3)$ & $11(1.6)$ \\
\hline \multirow[t]{5}{*}{ Anxiety/ Depression } & Level 1 & $68(64.8)$ & $421(69.9)$ & $489(69.2)$ \\
\hline & Level 2 & $34(32.4)$ & 157(26.1) & $191(27.0)$ \\
\hline & Level 3 & $0(0.0)$ & $11(1.8)$ & $11(1.6)$ \\
\hline & Level 4 & $1(1.0)$ & $4(0.7)$ & $5(0.7)$ \\
\hline & Level 5 & $2(1.9)$ & $9(1.5)$ & $11(1.6)$ \\
\hline
\end{tabular}

regardless of UF or non-UF group (Figs. 4 and 5). Figure 2 shows that the EQ-5D value was the lowest in the first gestational trimester regardless of UF status. However, this finding was not in agreement with results of some other published studies [30,31]. This lack of agreement might be caused by the policy of performing a full systemic prenatal examination and consultation in the second and third trimesters in China in addition to health-care appointments every 2 or 4 weeks, which might improve pregnant women's the HRQoL. Those with UFs had more health-related problems across a range of five dimensions (mobility, self-care, usual activity, pain/discomfort, and anxiety/depression) compared to those without (Table 4). About half of the pregnant women (46.7\%) suffered health problems associated with pain and discomfort; this was the greatest proportion of the five dimensions. Thus, it is necessary for the public healthcare system to focus on relieving this pain and discomfort when designing policies. 
Table 5 Ordered logistic regression analysis for each dimension in the EQ5D

\begin{tabular}{|c|c|c|c|c|}
\hline & & Odds ratio & $95 \% \mathrm{Cl}$ & $p$-value \\
\hline \multirow[t]{9}{*}{ Mobility } & Age & 0.98 & $0.93,1.02$ & 0.35 \\
\hline & $\mathrm{BMl}$ & 1.03 & $0.98,1.08$ & 0.26 \\
\hline & Local & 0.83 & $0.48,1.43$ & 0.50 \\
\hline & Gestation trimester & 1.77 & $1.21,2.60$ & $<0.01$ \\
\hline & Partner smoking & 1.10 & $0.64,1.87$ & 0.74 \\
\hline & Partner Alcoholics & 1.21 & $0.59,2.51$ & 0.60 \\
\hline & Gravidity & 1.17 & $0.95,1.45$ & 0.15 \\
\hline & Parity & 0.97 & $0.60,1.57$ & 0.90 \\
\hline & UF & 1.32 & $0.77,2.25$ & 0.31 \\
\hline \multirow[t]{9}{*}{ Self-care } & Age & 0.96 & $0.90,1.02$ & 0.17 \\
\hline & BMl & 1.00 & $0.94,1.08$ & 0.94 \\
\hline & Local & 1.15 & $0.48,2.76$ & 0.16 \\
\hline & Gestation trimester & 1.58 & $0.95,2.65$ & 0.08 \\
\hline & Partner smoking & 0.74 & $0.38,1.42$ & 0.36 \\
\hline & Partner alcoholics & 1.79 & $0.59,5.37$ & 0.30 \\
\hline & Gravidity & 0.91 & $0.64,1.28$ & 0.59 \\
\hline & Parity & 0.72 & $0.35,1.49$ & 0.38 \\
\hline & UF & 3.69 & $1.94,7.03$ & $<0.01$ \\
\hline \multirow[t]{9}{*}{ Usual activity } & Age & 0.98 & $0.93,1.03$ & 0.47 \\
\hline & BMl & 1.03 & $0.97,1.08$ & 0.34 \\
\hline & Local & 0.74 & $0.43,1.29$ & 0.29 \\
\hline & Gestation trimester & 1.38 & $0.94,2.00$ & 0.10 \\
\hline & Partner smoking & 1.46 & $0.80,2.66$ & 0.22 \\
\hline & Partner alcoholics & 1.16 & $0.54,2.49$ & 0.71 \\
\hline & Gravidity & 1.33 & $1.07,1.66$ & 0.01 \\
\hline & Parity & 0.56 & $0.33,0.94$ & 0.03 \\
\hline & UF & 2.11 & $1.25,3.55$ & $<0.01$ \\
\hline \multirow[t]{9}{*}{ Pain/Discomfort } & Age & 0.96 & $0.93,1.00$ & 0.06 \\
\hline & BMl & 1.00 & $0.96,1.04$ & 0.96 \\
\hline & Local & 2.16 & $1.26,3.71$ & $<0.01$ \\
\hline & Gestation trimester & 1.46 & 1.111 .93 & $<0.01$ \\
\hline & Partner smoking & 1.26 & $0.82,1.93$ & 0.29 \\
\hline & Partner alcoholics & 0.71 & $0.41,1.26$ & 0.25 \\
\hline & Gravidity & 1.07 & $0.90,1.29$ & 0.45 \\
\hline & Parity & 0.75 & $0.51,1.10$ & 0.14 \\
\hline & UF & 1.47 & $0.94,2.28$ & 0.09 \\
\hline \multirow[t]{4}{*}{ Anxiety/depression } & Age & 1.00 & $0.96,1.04$ & 0.93 \\
\hline & BMl & 1.01 & $0.97,1.06$ & 0.55 \\
\hline & Local & 1.07 & $0.65,1.78$ & 0.78 \\
\hline & Gestation trimester & 0.90 & $0.68,1.21$ & 0.49 \\
\hline
\end{tabular}

Table 5 Ordered logistic regression analysis for each dimension in the EQ5D (Continued)

\begin{tabular}{lllll}
\hline & Odds ratio & $95 \% \mathrm{Cl}$ & $p$-value \\
\hline Partner smoking & 0.85 & $0.56,1.31$ & 0.47 \\
Partner alcoholics & 0.97 & $0.53,1.76$ & 0.91 \\
Gravidity & 1.04 & $0.86,1.26$ & 0.68 \\
Parity & 0.60 & $0.39,0.91$ & $\mathbf{0 . 0 2}$ \\
UF & 1.18 & $0.74,1.89$ & 0.75 \\
\hline
\end{tabular}

Bold represented $p$-value $<0.05$

Besides, the EQ5D indices with respect to mobility and pain/discomfort dimensions fas reported by women without UFs were greater than those with UFs regardless of the trimester, which might be explained by lower levels of physical activities during the second and third trimesters [32]. Increased gravidity significantly correlated with the rising odds of usual activity problems (Table 5) because pregnant women who have increased gravidity in China mostly had their second child at advanced maternal ages because of the recent start of the Chinese two-child policy and long period of China's one-child policy. Under these conditions, pregnant women with advanced maternal ages were more likely to receive more medical care during pregnancy, which might be explain the increase in usual activity problems. In addition, decreased parity (mostly nulliparity) could contribute to the increase in usual activity problems. A previous study had clearly recognized a decrease in parity as a risk factor for the incidence of UFs [24], possibly because the production of estrogen and progesterone declines in parity and has considerable effects on fibroids' growth [33].

These findings offer additional and detailed evidence for the negative influence of UFs on HRQoL, which is in agreement with other cross-sectional studies. One web-based cross-sectional study investigated the HRQoL across 4848 women aged 18 to 49 years using the Uterine Fibroid Symptom-Quality of Life Questionnaire (UFS-QoL) and demonstrated a significant reduction in HRQoL in women with UFs [34]. An online cross-sectional study also found that the HRQoL might decrease with UFs and might be significantly impacted by UF-related symptoms [35]. Our study shows that UFs in pregnant women might affect the HRQoL scores in the self-care and usual activity dimensions (Table 5).

Comparative assessments of pregnant women with and without UFs based on gestational index and chronic conditions indicate that some of these independent factors contributed considerably to the incidence of UFs, while other conditions were in an inverse relation with UFs. Ages and BMIs are higher among those with UFs than those without, which can be explained by a greater age indicating more gravidity, abortion, and opportunities for pregnancy during 
advanced maternal age (Table 1) [1, 36]. The incidence of UFs $(p<0.05)$ will cause higher gravidity and abortion rates according to our data (Table 1), and this finding could be explained by the fact that UFs can significantly lead to more infertility [3]; therefore, gravidity and abortion rates will increase correspondingly. We were surprised about the self-report smoking status of the pregnant women as none of them smoked. This finding might be due to the family planning policy (one-child policy in the past and two-child policy recently) in China, which might increase mothers' concerns about their baby's health status although self-report bias is another possible explanation. Also, it seemed that there was no statistical significantly impact between partner smoker/alcohol consumption and pregnant women's HRQoL state in the different dimensions (Table 5).

One prior study demonstrated that the rate of Cesarean section has increased from $28.8 \%$ in 2008 to $34.9 \%$ in 2014 in China [37], and this rate was shown to be related to family income, education, health insurance, Chinese health policy, and cultural background, among other factors [38]. In pregnant women with UFs, Cesarean section rates reached $67.0 \%$, which was much higher than the rate in the normal population worldwide. Cesarean section may be the most suitable management for pregnant women with other pre-pregnancy conditions [39]. These findings are consistent with prior published data [35, 40-43].

Although there was no significant correlation between low HRQoL and poor clinical outcomes of pregnant women, further studies need to be done to verify these results. QoL is becoming an increasingly important indicator of the effectiveness of the medical intervention, and we should pay greater attention to QoL during pregnancy in our future practice.

\section{Limitations}

There were some limitations to this study. This was a cross-sectional study, and the data was obtained from an EQ-5D questionnaire in which there was relatively a subjective measurement of pregnant women's HRQoL. Thus, self-report bias may be the main bias in this study. This study design also presents limitation with respect to both non-response and volunteer biases. Furthermore, we do not make comparisons against different instrument other than the EQ-5D. There are some studies that have used the UFS-QoL to assess the HRQoL in pregnant women [34, 35]. This research could provide HRQoL data for pregnant women who were evaluated using the EQ-5D-5 L, and this information could be useful in cost-utility analyses in the healthcare-related economic area. Additionally, the HRQoL is an important indicator of a patient's overall state and plays an increasingly important role in evaluation in the clinic although there is no significant difference with respect to clinical outcomes between women with and without UFs in the short-term. Nevertheless, we believe that better quality life-related studies should be performed in order to further investigate the role of QoL in the clinic and monitor long-term effects on QoL. Future studies will need to use a cohort to observe the changes in HRQoL in pregnant women.

\section{Interpretation}

To our knowledge, this is the first clinical study to use the EQ-5D-5 L to focus on HRQoL during pregnancy in China and detect independent factors that could impact HRQoL in pregnant women with UFs. Also, this study shows that EQ-5D index may be a better index than EQ-VAS for pregnant women with uterine fibroids and possibly for other medical comorbidities or complications. Furthermore, the HRQoL data assessed by the EQ-5D-5 L could be used to perform cost-utility analyses in the future. The clinical outcomes of pregnant women with UFs could still offer insight for the clinical physician when considering the possibility of latent complications in pregnant women with UFs.

\section{Conclusion}

In this study, we evaluated the influence of UFs on HRQoL in pregnant women and found that the EQ5D assessment instrument outperformed the EQ-VAS. Our findings demonstrated that UFs significantly affected HRQoL in pregnant women in terms of the self-care and usual activity dimensions. Independent factors, such as living locally, gravidity and parity times, and gestational trimester, could have a significant impact on the HRQoL. Finally, whether clinical outcomes may affect HRQoL scores need to be precisely analyzed and requires further research.

\footnotetext{
Abbreviations

BMI: Body mass index; Cls: Confidence intervals; EQ-5D: The EuroQoL Group's five-dimension questionnaire; EQ-5D-3 L: The EuroQoL Group's fivedimension three-level questionnaire; EQ-5D-5 L: The EuroQoL Group's fivedimension five-level questionnaire; EQ-VAS: EuroQoL Group's visual analog scale questionnaire; HRQoL: Health-related quality of life; ORs: Odds ratios; PROM: Premature rupture of membrane; QoL: Quality of Ife; UFs: Uterine fibroids; UFS-QoL: Uterine Fibroid Symptom-Quality of Life; VAS: Visual analog scale
}

\section{Acknowledgments}

The authors are grateful to Yunyi Jian, Jingyan Zhai, Yu Cheng, Xianghao Cai, and Bangsheng Jiang for previous support in collecting data.

\section{Funding}

There is no financial support in this study.

\section{Availability of data and materials}

The datasets used and/or analyzed during the current study are available from the corresponding author on reasonable request.

\section{Authors' contributions}

WM contributed to the idea and design of the whole research process and contributed to the final version of the manuscript. HW contributed to the design and management of the whole study, data analysis, and final version 
of the manuscript. YW contributed to the hypothesis of study and final version of the manuscript. TM, YS, ZW, XH WS, TC, and YW contributed to data collection, data analysis, and final version of the manuscript. WD contributed to data collection and drafting the final version of the manuscript. HC and ZL contributed to data analysis and drafting the final version of the manuscript. ZW contributed to the design of the study, the guidance of research, and discussion of the final version of the manuscript. All authors read and approved the final manuscript.

\section{Ethics approval and consent to participate}

Ethical approval was granted by the Institutional Review Board of The First Affiliated Hospital of Sun Yat-sen University (ICE-2017-296).

\section{Consent for publication}

Not applicable

\section{Competing interests}

The authors declare that they have no competing interests.

\section{Publisher's Note}

Springer Nature remains neutral with regard to jurisdictional claims in published maps and institutional affiliations.

\section{Author details}

'Department of Obstetrics and Gynaecology, The First Affiliated Hospital of Sun Yat-sen University, Guangzhou, China. ${ }^{2}$ Pharmacoepidemiology and Pharmacoeconomic, Department of Medicine, Brigham and Women's Hospital and Harvard Medical School, Boston, MA, USA. ${ }^{3}$ School of Medicine, Jinan University, Guangzhou, China.

\section{Received: 12 September 2018 Accepted: 1 May 2019}

Published online: 24 May 2019

\section{References}

1. He Y, Zeng Q, Dong S, Qin L, Li G, Wang P. Associations between uterine fibroids and lifestyles including diet, physical activity and stress: a casecontrol study in China. Asia Pac J Clin Nutr. 2013;22(1):109-17.

2. Zhang W, Cheng Z, Qu X, Dai H, Ke X, Chen Z. Overexpression of myosin is associated with the development of uterine myoma. J Obstet Gynaecol Res. 2014:40(9):2051-7.

3. Vlahos NF, Theodoridis TD, Partsinevelos GA. Myomas and Adenomyosis: impact on reproductive outcome. Biomed Res Int. 2017;2017:5926470.

4. Jiang X, Thapa A, Lu J, Bhujohory VS, Liu Y, Qiao S. Ultrasound-guided transvaginal radiofrequency myolysis for symptomatic uterine myomas. Eur J Obstet Gynecol Reprod Biol. 2014;177:38-43.

5. Ertunc D, Uzun R, Tok EC, Doruk A, Dilek S. The effect of myoma uteri and myomectomy on sexual function. J Sex Med. 2009;6(4):1032-8.

6. Chambers GM, Randall S, Mihalopoulos C, Reilly N, Sullivan EA, Highet N, Morgan VA, Croft ML, Chatterton ML, Austin MP. Mental health consultations in the perinatal period: a cost-analysis of Medicare services provided to women during a period of intense mental health reform in Australia. Aust Health Rev. 2017;42(5):514-21.

7. Nicholson WK, Setse R, Hill-Briggs F, Cooper LA, Strobino D, Powe NR. Depressive symptoms and health-related quality of life in early pregnancy. Obstet Gynecol. 2006;107(4):798-806.

8. Shen TC, Yang CY, Huang YJ, Lin CL, Sung FC. Risk of depression in patients with uterine leiomyoma: a nationwide population-based cohort study. J Affect Disord. 2017;213:126-30.

9. Carreon LY, Bratcher KR, Das N, Nienhuis JB, Glassman SD. Estimating EQ-5D values from the neck disability index and numeric rating scales for neck and arm pain. J Neurosurg Spine. 2014;21(3):394-9.

10. Schaller A, Dejonghe L, Haastert B, Froboese I. Physical activity and healthrelated quality of life in chronic low back pain patients: a cross-sectional study. BMC Musculoskelet Disord. 2015;16:62.

11. Neumann PJ, Goldie SJ, Weinstein MC. Preference-based measures in economic evaluation in health care. Annu Rev Public Health. 2000;21:587-611.

12. Sarkin AJ, Groessl EJ, Carlson JA, Tally SR, Kaplan RM, Sieber WJ, Ganiats TG. Development and validation of a mental health subscale from the quality of well-being self-administered. Qual Life Res. 2013;22(7):1685-96.
13. Coyne KS, Margolis MK, Bradley LD, Guido R, Maxwell GL, Spies JB. Further validation of the uterine fibroid symptom and quality-of-life questionnaire. Value Health. 2012;15(1):135-42.

14. Bruggmann D, Louwen F, Braun T, Klingelhofer D, Bauer J, Bendels $\mathrm{MH}$, Bundschuh M, Quarcoo D, Jaque J, Wanke EM, et al. The uterine fibroid/ myoma tumour: analysis of the global research architecture using densityequalizing mapping. Reprod BioMed Online. 2018;36(2):227-38.

15. Gill TM, Feinstein AR. A critical appraisal of the quality of quality-of-life measurements. JAMA. 1994:272(8):619-26.

16. Herdman M, Gudex C, Lloyd A, Janssen M, Kind P, Parkin D, Bonsel G, Badia $X$. Development and preliminary testing of the new five-level version of EQ5D (EQ-5D-5L). Qual Life Res. 2011;20(10):1727-36.

17. Lee CF, Ng R, Luo N, Wong NS, Yap YS, Lo SK, Chia WK, Yee A, Krishna L, Wong $C$, et al. The English and Chinese versions of the five-level EuroQoL Group's five-dimension questionnaire (EQ-5D) were valid and reliable and provided comparable scores in Asian breast cancer patients. Support Care Cancer. 2013:21(1):201-9.

18. Luo N, Cheung YB, Ng R, Lee CF. Mapping and direct valuation: do they give equivalent EQ-5D-5L index scores? Health Qual Life Outcomes. 2015;13:166.

19. McCaffrey N, Kaambwa B, Currow DC, Ratcliffe J. Health-related quality of life measured using the EQ-5D-5L: south Australian population norms. Health Qual Life Outcomes. 2016;14(1):133.

20. Devlin NJ, Brooks R. EQ-5D and the EuroQol group: past, present and future. Appl Health Econ Health Policy. 2017;15(2):127-37.

21. Feng $Y$, Parkin D, Devlin NJ. Assessing the performance of the EQ-VAS in the NHS PROMs programme. Qual Life Res. 2014;23(3):977-89.

22. Lean SC, Derricott H, Jones RL, Heazell AEP. Advanced maternal age and adverse pregnancy outcomes: a systematic review and meta-analysis. PLoS One. 2017;12(10):e0186287.

23. Chiaffarino F, Ricci E, Cipriani S, Chiantera V, Parazzini F. Cigarette smoking and risk of uterine myoma: systematic review and meta-analysis. Eur J Obstet Gynecol Reprod Biol. 2016;197:63-71.

24. De La Cruz MS, Buchanan EM. Uterine fibroids: diagnosis and treatment. Am Fam Physician. 2017;95(2):100-7.

25. Fernandez A, Saameno JA, Pinto-Meza A, Luciano JV, Autonell J, Palao D, Salvador-Carulla L, Campayo JG, Haro JM, Serrano A, et al. Burden of chronic physical conditions and mental disorders in primary care. Br J Psychiatry. 2010:196(4):302-9.

26. van Hout B, Janssen MF, Feng YS, Kohlmann T, Busschbach J, Golicki D, Lloyd A, Scalone L, Kind P, Pickard AS. Interim scoring for the EQ-5D-5L: mapping the EQ-5D-5L to EQ-5D-3L value sets. Value Health. 2012;15(5): 708-15.

27. Mansikkamaki K, Raitanen J, Malila N, Sarkeala T, Mannisto S, Fredman J, Heinavaara S, Luoto R. Physical activity and menopause-related quality of life - a population-based cross-sectional study. Maturitas. 2015;80(1):69-74.

28. Kind P, Dolan P, Gudex C, Williams A. Variations in population health status: results from a United Kingdom national questionnaire survey. BMJ. 1998; 316(7133):736-41

29. Sun $\mathrm{S}$, Chen J, Johannesson M, Kind $P, X u$ L, Zhang $Y$, Burstrom $K$. Population health status in China: EQ-5D results, by age, sex and socioeconomic status, from the National Health Services Survey 2008. Qual Life Res. 2011;20(3):309-20.

30. Da Costa D, Dritsa M, Verreault N, Balaa C, Kudzman J, Khalife S. Sleep problems and depressed mood negatively impact health-related quality of life during pregnancy. Arch Womens Ment Health. 2010;13(3):249-57.

31. Tendais I, Figueiredo B, Mota J, Conde A. Physical activity, health-related quality of life and depression during pregnancy. Cad Saude Publica. 2011; 27(2):219-28.

32. Santos PC, Abreu S, Moreira C, Santos R, Ferreira M, Alves O, Moreira P, Mota J. Physical activity patterns during pregnancy in a sample of Portuguese women: a longitudinal prospective study. Iran Red Crescent Med J. 2016; 18(3):e22455.

33. Pavone D, Clemenza S, Sorbi F, Fambrini M, Petraglia F. Epidemiology and risk factors of uterine fibroids. Best Pract Res Clin Obstet Gynaecol. 2018;46: 3-11.

34. Soliman AM, Margolis MK, Castelli-Haley J, Fuldeore MJ, Owens CD, Coyne KS. Impact of uterine fibroid symptoms on health-related quality of life of US women: evidence from a cross-sectional survey. Curr Med Res Opin. 2017;33(11):1971-8.

35. Wise LA, Palmer JR, Harlow BL, Spiegelman D, Stewart EA, Adams-Campbell $\mathrm{LL}$, Rosenberg L. Risk of uterine leiomyomata in relation to tobacco, alcohol 
and caffeine consumption in the black Women's health study. Hum Reprod. 2004;19(8):1746-54.

36. Dalton-Brewer N. The role of complementary and alternative medicine for the Management of Fibroids and Associated Symptomatology. Curr Obstet Gynecol Rep. 2016;5:110-8.

37. Li HT, Luo S, Trasande L, Hellerstein S, Kang C, Li JX, Zhang Y, Liu JM, Blustein J. Geographic variations and temporal trends in cesarean delivery rates in China, 2008-2014. JAMA. 2017;317(1):69-76.

38. Feng $\mathrm{XL}$, Xu L, Guo Y, Ronsmans C. Factors influencing rising caesarean section rates in China between 1988 and 2008. Bull World Health Organ. 2012:90(1):30-9 39A.

39. Sevket O, Ates S, Molla T, Ozkal F, Uysal O, Dansuk R. Hydrosonographic assessment of the effects of 2 different suturing techniques on healing of the uterine scar after cesarean delivery. Int J Gynaecol Obstet. 2014;125(3): 219-22.

40. Wise LA, Laughlin-Tommaso SK. Epidemiology of uterine fibroids: from menarche to menopause. Clin Obstet Gynecol. 2016;59(1):2-24.

41. Vilos GA, Allaire C, Laberge PY, Leyland N, Special C. The management of uterine leiomyomas. J Obstet Gynaecol Can. 2015;37(2):157-78.

42. Laughlin SK, Schroeder JC, Baird DD. New directions in the epidemiology of uterine fibroids. Semin Reprod Med. 2010;28(3):204-17.

43. Naftalin J, Jurkovic D. The endometrial-myometrial junction: a fresh look at a busy crossing. Ultrasound Obstet Gynecol. 2009;34(1):1-11.

Ready to submit your research? Choose BMC and benefit from:

- fast, convenient online submission

- thorough peer review by experienced researchers in your field

- rapid publication on acceptance

- support for research data, including large and complex data types

- gold Open Access which fosters wider collaboration and increased citations

- maximum visibility for your research: over $100 \mathrm{M}$ website views per year

At BMC, research is always in progress.

Learn more biomedcentral.com/submissions 